\title{
Video Game Escapism During Quarantine
}

\author{
Eian Prinsen ${ }^{1}, \&$ Damian Schofield ${ }^{1}$ \\ ${ }^{1}$ Department of Computer Science, State University of New York at Oswego, New York, USA \\ Correspondence: Damian Schofield, Department of Computer Science, State University of New York at Oswego, \\ New York, 13126, USA.
}

Received: August 17, 2021

Accepted: October 4, 2021

Online Published: October 12, 2021

doi:10.5539/cis.v14n4p36

URL: https://doi.org/10.5539/cis.v14n4p36

\begin{abstract}
Video games, and especially roleplaying games offer a way for players to escape stress and cope with stressors. Video games are a new, and potentially unique, mechanism for telling stories. They allow a player to not only interact with a world, but to fully immerse themselves in a digital world. Being able to quite literally escape to a fantasy world where your actions matter, and you're given control can be a great form of self-therapy for players.

This study aims to examine the results of a global pandemic and quarantine on player motivations and the reasons they play video games. The study collected detailed information on the video games genres and play time of each user and how it has been affected during self-isolation. Participants were also asked a series of questions that required them to evaluate their gaming habits both prior to and during the pandemic. This information was analysed to attempt to further understand players choices, in particular during a pandemic where there is an increased need to alleviate stress and experience a sense of escapism.
\end{abstract}

Keywords: video games, escapism, stress, player motivation, pandemic

\section{Introduction}

The use of storytelling, whether through theatre, books or movies, has been a form of escapism utilized by civilizations throughout history. Stories are an important part of human culture as are able to share lessons learned and perhaps explain the unknown. Stories allow viewers to learn from the experience of others while escaping from the troubles of their own lives. Because of this, many societies have curated a collection of books, plays and more recently movies, that have impacted the lives of the individuals who enjoy them, becoming important and even essential parts of culture.

Video games are a new, and potentially unique, mechanism for telling stories. They allow a player to not only interact with a world, but to fully immerse themselves in a digital world. Similar to many other forms of media, video games have a plethora of different genres to pull from, but unlike other media types, these genres can change the play style along with the narrative themes and elements.

Roleplaying games (RPGs) are a specific game genre in which players are given the ability to explore, interact and even alter the virtual world they inhabit. Roleplaying games pull heavily from broader fantasy tropes of the genre, based on works of authors such as J.R.R Tolkien, Robert E. Howard, George R.R. Martin, and H.P Lovecraft. While the game narratives can take place in a variety of settings, ranging from high fantasy to space opera, the ability to make decisions and explore are key elements of this game genre.

Over the past 20 years, many RPGs have tried to emulate popular, social, cooperative games like Dungeons and Dragons. Massively Multiplayer Online Role-Playing Games (MMORPG), such as World of Warcraft, allow the player to customize an avatar in which they can interact with the world co-operatively or competitively with other players (Blasi et al., 2019). This social aspect of MMORPGs can enhance the players engagement, immersion, and satisfaction with the game.

Video games are a unique form of entertainment because they challenge the player to "act and explore" within the virtual world, rather than act as a passive media observer Because of this, video games are often considered a form of active escapism, an interactive media that fully immerses the individual (Calleja, 2010).

One of the ways video games can reduce stress is by creating a sense of empowerment, within the game world. This sense of empowerment can provide profound benefit to the psychological health of players, and help to reduce anxiety (Kuo et al., 2016). 
Video games, in particular RPGs, are often associated with a sense of escapism, a way to help video game players relax and forget 'real world' concerns. Escapism is often defined as the act of seeking distraction from stressful situations in reality through the use of fantasy and entertainment. When a player engages in escapism, individuals often report a psychological state of mental absorption where their conscious mind is overtaken by the focal stimuli, in this case a video game (Russell et al., 2004).

\subsection{Exploring Escapism}

Most research work that has investigated escapism generally defines the concept in two different forms, active and passive. While both forms of escapism share the same function, the utility of both varies, and depending on the individual, either one can be more or less effective than the other.

- Active escapism is defined as the "Participative collaboration between the consumer and the marketing entity". A good example being video games, where the player is required to actively participate in order to progress the story (Mathwick et al., 2001).

- Passive escapism is defined as "Experiences in which consumers act only as observers through comprehension and appreciation". This can be summarized as any media that a person absorbs without the ability to interact or control the experience (Mathwick et al., 2001).

One of the key components of escapism is immersion, or the ability for an individual to mentally connect to the world with which they are engaged. The level of absorption and engagement that occurs while watching a movie or reading a novel will depend upon the degree to which a reader or viewer is captivated by the story, meaning that dull moments can easily break their sense of immersion.

Although both passive and active modes of escapism can provide immersion, passive sources of escapism don't provide an individual with a level of interactivity that is provided by active escapism (Kuo et al., 2016). This ability to interact with the world and the constant need for the player to actively progress the story can greatly affect a player's sense of immersion allowing the player to remain in an escapism mindset.

Even in a passive media source, such as a movie, a viewer can experience escapism through narrative transportation. It is important to note that not all instances of narrative transportation will qualify as escapism however, as both transportation and wish-fulfillment define that experience. Media that contains depressing or stressful content are likely to increase negative feelings for the individual. This can occur in roleplaying games like Dark Souls (Namco Bandai Games, 2011) that include elements of horror, and attempt to provide immersion by continually challenging the player (Kuo et al., 2016).

Active escapism has the ability to extend a video game player beyond both transportation and immersion by offering interactivity to complement the narrative and sensory engagement (Kuo et al., 2016). However, this advantage can also be a problem with interactive media, as some game mechanics can become disengaging for a player. Often players will lose any sense of immersion or escapism if they find that a gameplay loop is too tedious, or that there is a mismatch between the narrative structure and the gameplay.

Many game developers have, however, learned to take advantage of a number of tools to improve levels of escapism in their players. These tools include gameplay loops, hierarchical achievement goals, and narrative tropes. Other forms of more traditionally passive media have started to absorb these concepts in order to keep users engaged. Television shows have introduced the idea of allowing the viewer to make decisions that affect the outcome of the story, while cell phone applications and websites application have introduced gamification mechanics to keep users engaged.

\subsection{Roleplaying Games}

The virtual worlds created for roleplaying video games are unparalleled in their ability to rapidly immerse players and provide hitherto unachievable levels of escapism. A number of these games have developed online communities, even allowing players, and aspiring game developers, to add modifications (mods) to the game changing how the virtual world looks and even interacts with the player.

A good example of a single player roleplaying game is Skyrim (Bethesda Softworks, 2011). This game allows the player to make multiple important choices at the start of the game, opening up a new entire world to the player almost immediately. These choices allow the player to project their ideal self into a virtual world that they can control (Blasi et al., 2019).

In the real world an individual may sometimes feel a lack of perceived control when dealing with their identity. This can often occur when a person doesn't always see their real self as they ideally would like to be perceived. Research has shown that roleplaying video games can help give back this sense of control to a player, although 
in a temporary intangible state (Kuo et al., 2016).

Research has shown that this feeling of control is often a critical element in what drives an individual to seek escapism, video games offer a player more control than most other sources of media. This is particularly true for roleplaying games, since they allow a player to choose who they want to be within the game and gives them a high level of control over the game character's choices (Kuo et al., 2016).

\subsection{Gaming Motivations}

Many studies have been conducted to try to find links between the personality traits of an individual and their motivation to play specific genres/types of video games. Although the research has generally found that personality traits do not seem to predict specific gaming behavior when it comes to play time and genre, the findings do suggest that personality traits can be relevant to the reasons why a person might value particular forms of gaming (Park et al., 2011; Yee et al., 2012; Normal et al., 2014).

This implies that players find themselves relying on certain video games mainly due to external stressors and factors, and that this behaviour is not solely based on personality. Additionally, studies have found that player age has a positive association with escapism as a motivation for playing online games. This suggests that as an individual's life burdens and responsibility increase, they may become more motivated to escape from their external stressors (Park et al., 2011). This idea can be reflected onto most forms of escapism for adults, as hobbies become ever more important as an individual gets older.

A recent study found correlations between stress relief and active escapism. The study focused on emotional coping mechanisms and found that active escapism was more often motivated by the desire to regulate negative affect which is provided through emotion focused coping (Kuo et al., 2016). Other studies have found that players with emotion focused coping mechanisms are more likely to use games to help alleviate stress than those who rely on problem focused coping styles. This makes sense as those with problem focused coping styles often look for a solution to their problems. Video games provide no solution for real world problems but can potentially provide the escape emotion focused individuals desire (Bowditch et al., 2018). However, using video games as a form of escape can potentially create problems, since players are spending time escaping from their issues less time is spent dealing with the issue, which can lead to issues never being resolved. This method of coping was found to not be repressive, but rather a conscious motivated choice by the player (Kuo et al., 2016).

Studies have also found that some players find themselves motivated by pressures that come from a sense of self-worth attached to game activity. This suggests that engagement in gaming activity can sometimes be an attempt to prove oneself (Mills et al., 2017). If an individual isn't recognized by their achievements in real life, whether that be by others or themselves, they are more likely to rely on their virtual accomplishments. Some players may attach self-esteem to video games, this could indicate that players who are living through a stressful situation or struggling with mental health issues will rely on these virtual achievements to validate their self-worth (Mills et al., 2017). This can become problematic and lead to greater issues of depression and anxiety, all of which are often seen as symptoms of game addiction.

\subsection{Gaming Addiction}

Many people often find themselves relying on external and controllable habits to help them cope with life. These include dangerous habits like binge eating, drug use and gambling, which are often noted as being a form of escapism from real world trauma. However, these forms of escapism lack the narrative structure offered by video games (roleplaying video games in particular) that can create a more focused form of escapism rather than acting solely as a coping mechanism (Kuo et al., 2016).

Video game addiction is a relatively new problem in society but is generally regarded as a fairly serious issue (Griffiths et al., 2012). Games are usually designed as an easy way for players to pass time and to find relief from everyday problems. Video games bring exciting visual elements, engaging stories, active engagement and the ability to play online with others, all into a single medium. Video games are the most widespread example of the use of active escapism, as most other sources of media allow little to no interaction. Since roleplaying video games create an alternate world with a new character for the player to project themselves onto, this can become more enticing to players in times of high stress.

However, studies have shown that playing video games for escapist reasons is often associated with strong belief that the digital world was as realistic as their real one leading to a decrease in their support and interest in real world problems. This belief, coupled with the stationary nature of gaming, can lead to a reduction in the players wellbeing (Kaczmarek \& Drążkowski, 2014). 
Studies on World of Warcraft (Blizzard Entertainment, 2004) have found that individuals with high emotion dysregulation were more likely to show problematic involvements in online roleplaying games. Emotion dysregulation helped to predict both escapism motives and problematic gaming signs in players. These results were consistent with other research showing that World of Warcraft players who have difficulty with emotion regulation can increasingly develop symptoms such as depression and anxiety (Blasi et al., 2019).

Using video games as a coping mechanism rather than addressing real world issues can also lead to further problems for an individual, and without a way to regulate consumption of media, the individual might find themselves in a worse mental state than before. Research suggests that escapist motivations in emotionally dysregulated individuals can lead to an increase in problematic gaming. Due to the immersive nature of the experience, players struggling with mental health may become reliant on games (Chiu et al., 2004; Blasi et al., 2019).

While the relationship between escapism and negative outcomes in gaming have been established multiple times, some studies found that escapism alone does not always lead to adverse effects. Players who also have a strong problem focused style of coping with their everyday stressors were not only associated with fewer negative outcomes, but the escapism helped to protect these individuals against more negative gaming habits (Bowditch et al., 2018). This suggests that players who use video games to escape are less likely to see negative outcomes, such as addiction or depression, if they continue to address real world issues too. The use of problem-focused coping actually helps to regulate the negative outcomes of gaming that can be found in emotion focused coping (Chiu et al., 2004; Bowditch et al., 2018).

Video games are often compared to gambling, when addiction is referenced, and both have been under scrutiny in the public eye for their possibly negative and addictive nature (Wood \& Griffiths, 2007). Both industries have learned from each other, casinos have long used gamification tactics to help lure in perspective players and game developers have started to introduce loot boxes into their games as a way to earn extra money from players. However, a number of studies have shown that gambling can be an effective means of coping with external stressors, perhaps again by offering a form of escapism for the player. Gambling and the concept of chance has been also been found to be an effective tool in altering the mood of players, suggesting that the thrill of winning big is enough to give temporary relief to players (Wood \& Griffiths, 2007).

\section{Method}

Natural catastrophes have a habit of creating societies that search for comfort during this time of need. A pandemic is no different from other forms of disaster in this aspect, however it does create a unique situation in which most of society spends their time indoors during periods of quarantine. During the age of the internet, people lean on social experiences through any means possible and utilized the technology given to create new forms of comfort. Research has repeatedly shown that during times of disasters, a populations media consumption habits change significantly (Adams and Schofield, 2020).

This study aims to examine the results of a global pandemic and quarantine on player motivations and their motivations to play video games. The study collected detailed information on the video games genres and play time of each user and how it has been affected during self-isolation. Participants were also asked a series of questions that require them to evaluate their gaming habits both prior to and during the pandemic. This information will be analysed to attempt to further understand players choices, in particular during a pandemic where there is an increased need to alleviate stress and experience a sense of escapism.

Hypothesis 1: People will increase the time spent playing video games.

Hypothesis 2: People will try new genres of video games.

Hypothesis 3: People are more likely to try to find a console during quarantine.

\subsection{Participants}

The study used 32 participants whose age ranged from 20 to 40 years old. The participants were recruited from gaming based social media pages to ensure a general interest in video games and were required to own a device capable of playing videos games (console, personal computer or phone). The range of participant demographics meant that there was a significant distribution of game genres, play times and gaming history. The participant pool was limited due to ongoing pandemic restrictions at the time the experiment was undertaken. 


\subsection{Metrics}

All participants were asked to take Yee's Gamer Motivation test prior to the survey (Yee et al., 2012) ${ }^{1}$. The survey then asked the participants to list their top three traits according to Yee's Gamer Motivation. The survey then asked participants to give general information about the gaming platforms they used, preferred genres and what they saw as important elements in the video games they played. Additionally, the survey asked the participants to reflect on their gaming habits from before the pandemic compared to during the pandemic. The survey ended with a few open-ended questions asking the participants favorite games, as well as games they may have started playing during the pandemic.

\subsection{Procedures}

Participants were sent a link to the survey which first redirected them to take Yee's Gamer Motivation survey. After completing the preliminary survey, each participant was asked to enter some of the information provided from the motivation survey. Following the preliminary survey, participants were then directed to reflect on their gaming habits both before and during the pandemic. This was directed by a series of 15 questions both multiple choice and open ended.

\section{Results}

This experiment took place during the summer of 2020 in the height of the global Covid-19 pandemic. This meant that the experiment was conducted fully online, while most participants were in quarantine. Data was collected from 32 participants who were recruited through email, group chats, social media and word of mouth. The participant gender split was slightly uneven with 17 men and 15 women.

Participants were asked to give an estimate of the time each week spent playing games both prior to and during the Covid-19 pandemic. The results of the survey show that 18 out of our 32 participants identified that they have seen an increase in their weekly play time since the start of quarantine (Figure 1). Out of the remaining 14 participants none of them saw a decrease in playtime, and only one of them identified with the maximum play time provided by the survey (more than 40 hours) for both questions.

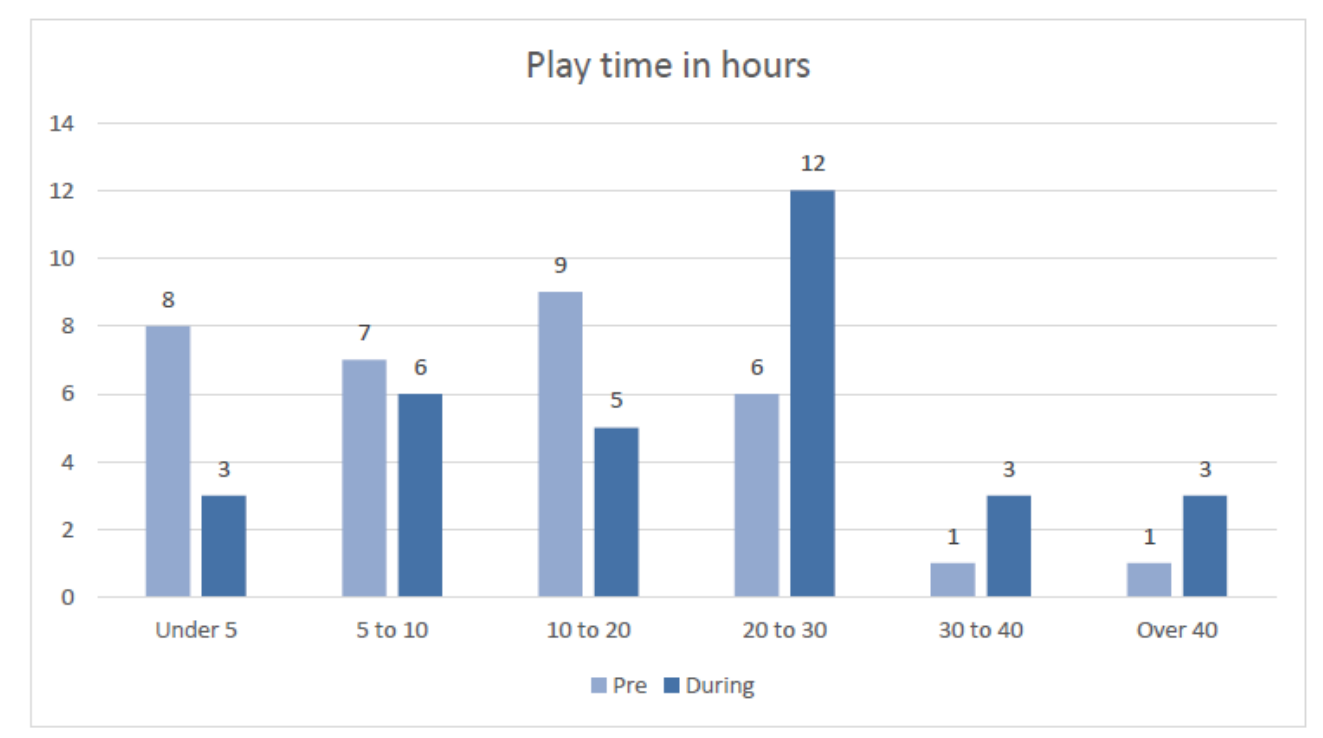

Figure 1. Playtime prior to and during Covid-19

The top three choices (participants who played over 20 hours per week) all doubled in size during the quarantine period. Keeping in mind the other hobbies and activities that some participants may have undertaken during their newfound free time, these results are significant

Figure 2 also shows that if reported gender is considered, women answered more often that they saw an increase in play time (10 out of 15 increased), whereas men remained more consistent ( 8 out of 17 increased). The male participants had a higher average play time overall, but most of the women surveyed had found an increased interest in video games during the quarantine. Additionally, when asked how often the participants found

\footnotetext{
${ }^{1}$ The Yee Gamer Motivation Survey: https://apps.quanticfoundry.com/surveys/start/gamerprofile/
} 
themselves playing games, more women answered "Everyday" or "Weekdays" versus men who answered, "When I find time" and "Weekends". When asked what games they found themselves playing, most of these women answered with games like Animal Crossing, Stardew Valley, and other light RPGs. These games reward short play times and encourage the player to check in more frequently.

The survey also asked participants to list their top three video game genres and included a list of the eleven most popular genres. Roleplaying games received the highest votes with 25 of the 32 participants choosing it as one of their favorite genres. Shooter games followed right behind with 16 of the participants choosing them, followed then by 12 of the participants choosing survival games (Figure 3).

The difference between favorite genre between men and women is not significant, however a few trends are apparent that might prove significant if a larger pool of participants was used. The data provided an indication that men prefer competitive games (Call of Duty, Super Smash Bros, Street Fighters) and Online games (World of Warcraft, Guild Wars), where women play mostly single player games (Skyrim, Animal Crossing, Zelda). Additionally, more of the men showed interest in the same mainstream games as one another, whereas more of the female participants played unique titles and retro games.

This experiment did not collect data that went into the reasons for these gender-based differences. However, other research by the authors has collected data that, to some degree explains these trends (Adams and Schofield, 2020).

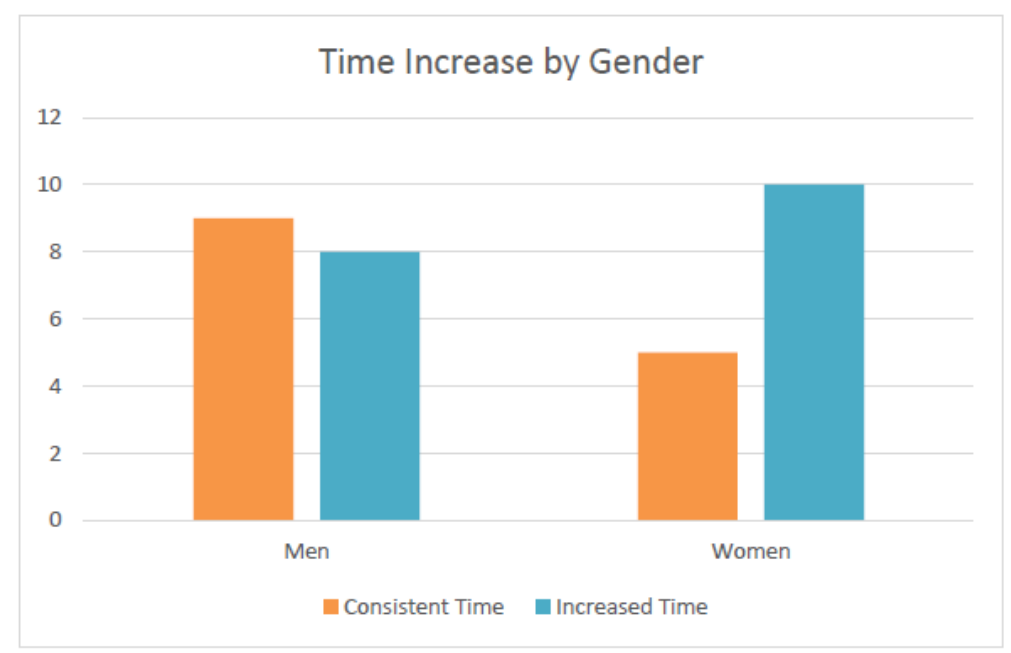

Figure 2. A comparison between time spent

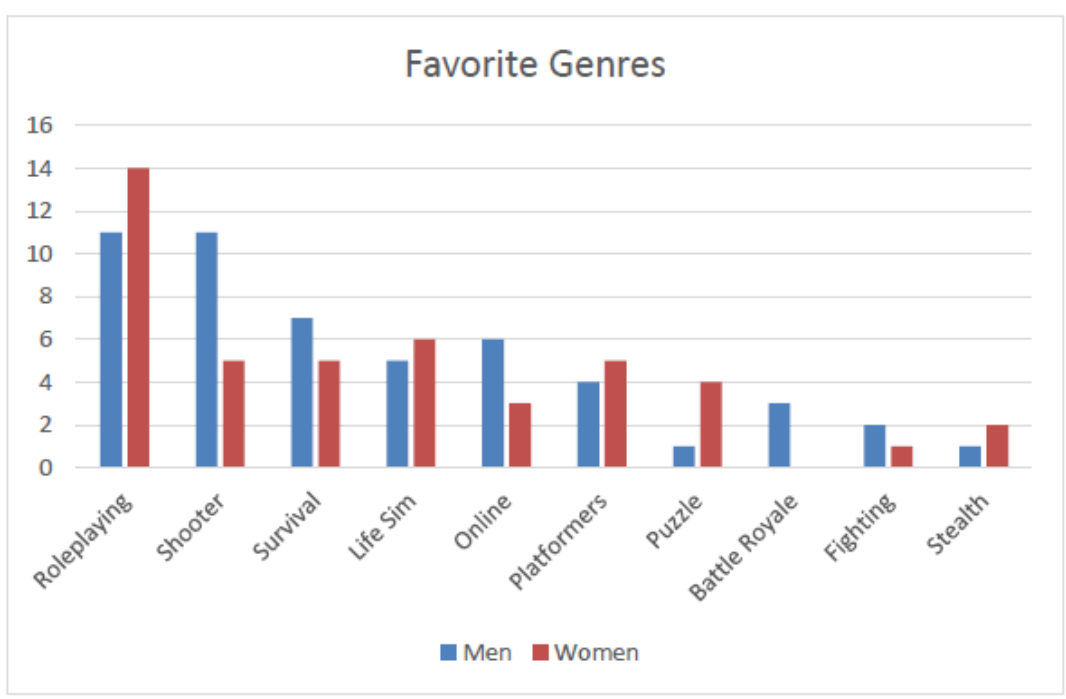

Figure 3. Favorite genres between men and women

Reflecting on this, the participants were asked to gauge their interest in both roleplaying and life simulation 
games prior to the pandemic. Unsurprisingly, 20 out of the 32 participants stated that they were very interested in the roleplaying genre, whereas the life simulation genre received only 13 likes and a few dislikes (Figure 4). The participants were also given a text field to enter some of the games they had played during quarantine. The most popular answers were almost all roleplaying games, or games with roleplaying elements. Some examples of these games included World of Warcraft, The Legend of Zelda, Fallout and Skyrim.

Participants were also asked to reflect on what has motivated them to play during quarantine. The responses show that most participants were looking to achieve some level of escapism, and to a lesser extent fill their newfound free time. Socialization and boredom also prove to be strong motivators for participants playing video games. These results support the predictions that participants would be looking to fill voids created by the quarantine, namely lack of socialization and an abundance of free time, over other motivations such as competition and accomplishment (Figure 5).

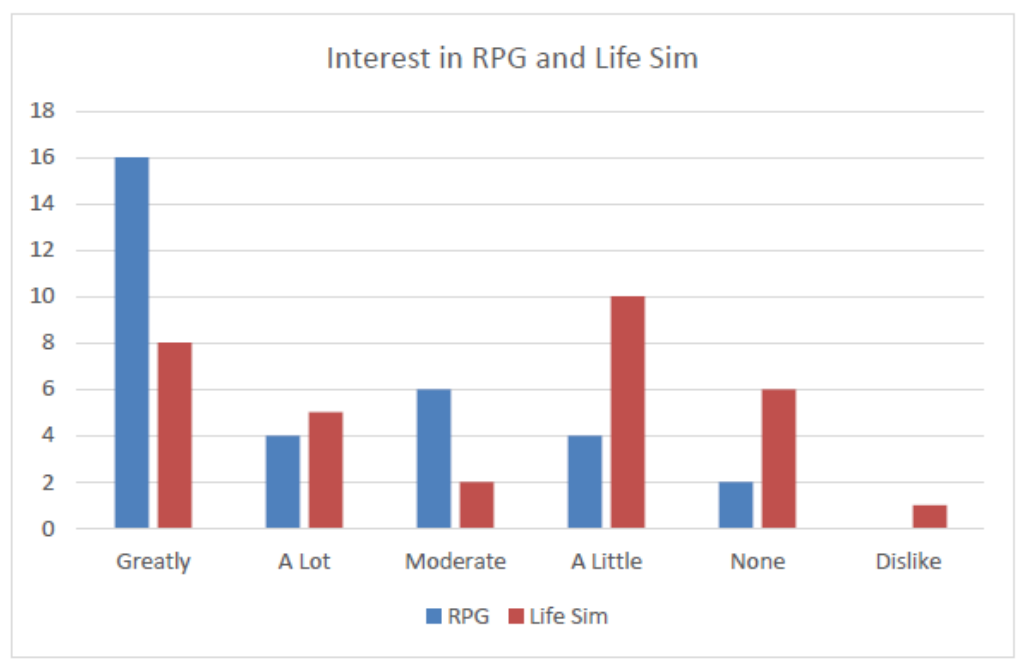

Figure 4. Participant interest in RPG and Life Sim

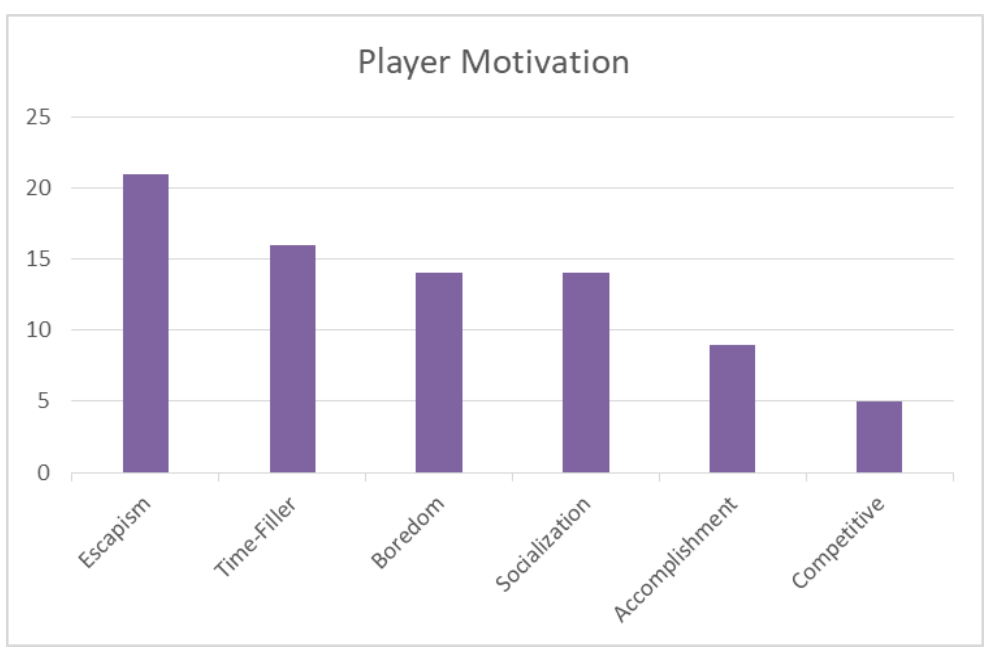

Figure 5. Motivation of players during quarantine

Other data gained from the survey remained evenly split and mostly non-significant. For example, the survey asked each participant to list preferred gaming consoles. The results split fairly evenly with many participants owning multiple consoles, mobile devices and computers. The highest ranked category was mobile gaming, with 28 of the 32 participants playing on either a mobile phone or a handheld gaming console like the Nintendo 3DS and Nintendo Switch. Since most adults own a mobile phone it is hard to draw any significant conclusion from this result. Excluding mobile phones from the analysis and only counting for handheld consoles, mobile gaming still remains the top category with 26 participants owning a Nintendo Switch.

Mobile devices and personal computers were favored over their console counterparts for the participants. Out of 
the 32 participants surveyed 26 owned a Nintendo Switch followed closely by personal computers with 25 . Current generation consoles fell behind with 11 for the PlayStation 4, and 7 for the Xbox One. Retro consoles and older handhelds also sat near the bottom of the group (Figure 6).

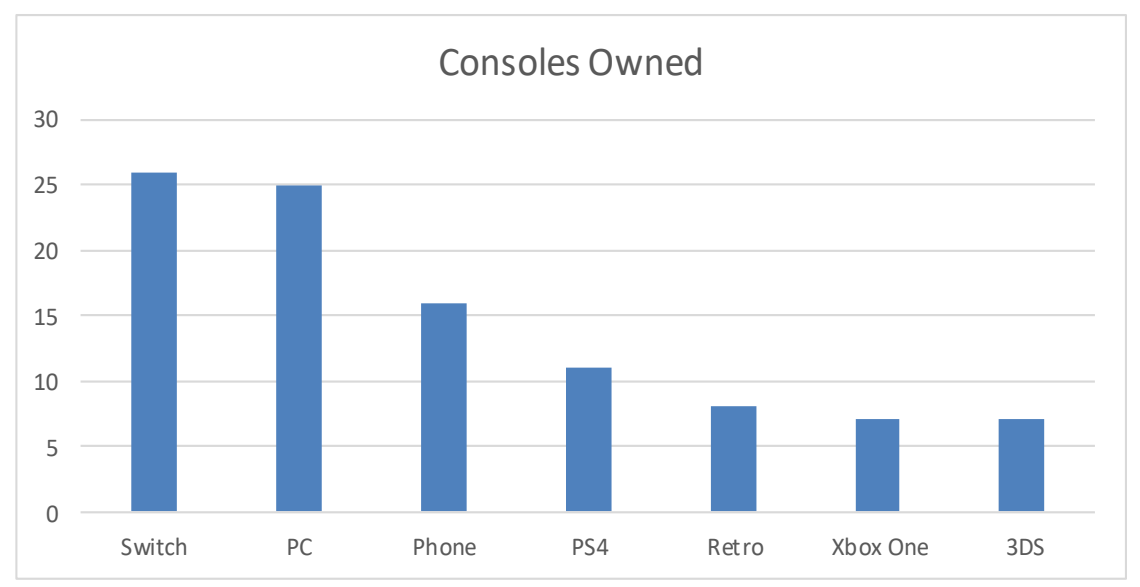

Figure 6. Consoles owned by the participants

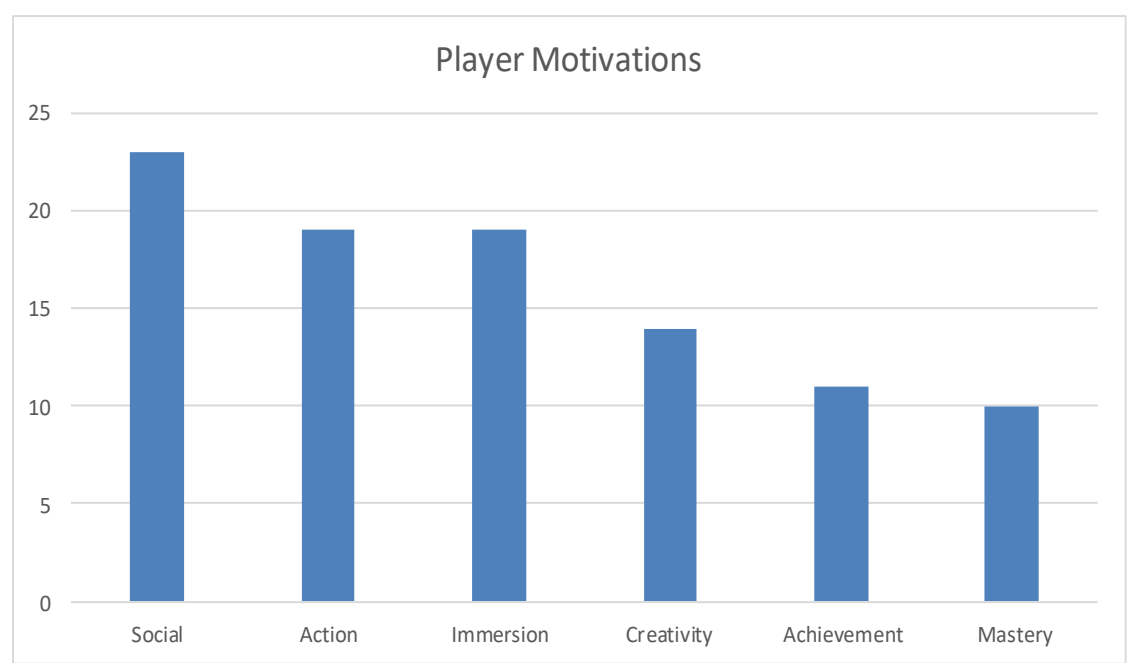

Figure 7. Gamer motivations according to Yee et al. (2012)

Additionally, the participants were asked whether they bought any new game devices during the pandemic. Only 6 of the 32 participants purchased a new device during the pandemic. This number could have been affected by a shortage in consoles and appropriate hardware that developed due to the pandemic.

\section{Analysis}

In addition to the data collected on play time, console availability and other qualitative information, participants were also asked to take Yee's survey on gamer motivation and share their results. The survey asks the participant a series of questions that upon completion determines their motivations as players across six categories. The player motivation categories are social, action, immersion, creativity, achievement, and mastery (Figure 6). This study asks the participant to list their top three motivations for playing according to Yee's survey and these results were then correlated with the previous data collected.

The results from Yee's survey showed a strong tend towards socialization, with 23 participants having this motivation in their top three, and mastery received the least results with only 10 participants reporting this motivation. These results test a participant's general interests when gaming without a self-evaluation bias and without taking into account any influence of a pandemic quarantine.

It appears that the majority of the participants primarily use gaming as a social outlet while more 
time-consuming elements like Achievements and Mastery are less interesting to them. Predictably, when these players were asked what type of games they prefer to play, almost all social gamers answered either Online Social or Competitive. A few social players did answer that they preferred single player experiences. However, when asked to list games they've enjoyed during the quarantine, every outlier answered with at least one game that focuses on social interactions (Animal Crossing, Stardew Valley, Sims). While these players prefer to play without interacting with other players, they instead enjoyed games that require the player to socialize with non-player characters.

The group of participants who did not tend towards socialization from Yee's survey all share similar traits. Each of the remaining 9 participants had either Creativity or Immersion as a trait, most of them having both. Additionally, when asked what has motivated them to play while under quarantine, all of them stated Escapism as a primary reason. This isn't an expected result, since escapism was the most common answer to the question. However, this escapism result is also supported by not only Yee's survey, but also the games each participant listed. Of the 9 participants who did not receive socialization as a primary trait, each of them listed one or more roleplaying game as a favorite title. This suggests that participants who were not identified as socially driven gamers, all share a commonality in using roleplaying games as a form of escapism. Since roleplaying games allow for a higher level of immersion and creativity, due to the ability to create and project yourself onto a fictional character, these participants preferred this outlet to their social counterparts.

Another correlation can be made between the Mastery and Achievement traits when compared to total play time. During quarantine, almost all the participants who were labeled with this trait, identified that they played more than the others, about 20 to 50+ hours, a week. This is likely due to the amount of time games require for players to learn and master mechanics, and the amount of time required to $100 \%$ complete games. To support this, most participants in this group stated that they prefer competitive games with a high skill ceiling (Valorant, League of Legends, Monster Hunter) or games that provide the player with a lot of content and collectibles (MMORPG's, Fallout, Last of Us). Both of these traits have a higher time commitment in order to succeed and reach successive areas of the game.

The results presented by Yee's player motivation survey don't necessarily align with the participants self-assessment of what they are looking for in a new game. Participants were asked why they played certain games, and given choices such as challenge, relaxation, social and adventure. Over half of the participants chose adventure as the most important feature. This may support the need for escapism for the majority of the participants, as it allows them to ignore their own problems in favor of the problems of a fantasy world and characters. These traits do not function in isolation, and it's apparent that these traits are more effective when working together. Before video games had become story telling devices, games like Dungeons and Dragons were used as a social tool for players to escape into, similar to the modern MMO. Although video games have grown and changed, those who play them appear to still have the same needs.

\section{Conclusions}

Video games, roleplaying games in particular, offer a potential mechanism for players to escape real-world issues and cope with stressors. The ability to escape into a fantasy world where actions matter, and players are given control can be a great form of self-therapy. Loss of perceived control in the real world is often the driving point for stress, and video games have the ability to return some sense of control to the player (Kuo et al., 2016).

Studies have found that needs frustrations, defined as the extent to which an individual feels obstructed in their pursuits of satisfaction in their daily life can be a strong predictor of behavioral addictions, such as game addiction (Vansteenkiste \& Ryan, 2013). The research demonstrated that obstruction of one's self-worth, defined through needs frustrations, can be major factor when predicting maladaptive outcomes like depression (Mills et al., 2017). While video games often to be a valuable tool in helping to restore a player's self-esteem, they can occasionally provide a mechanism that can lead to the development of serious psychological issues.

The work reported in this paper demonstrated the importance of escapism to many video games players. Although these games can often be useful in escaping from issues in the player's life, they do not solve the real issue. The experimental data in this project correlated the time spent gaming with game genres and player motivations. However, studies have shown that longer gaming periods are also often associated with individuals who are looking to cope with emotions, rather than those who are more problem focused (Wood \& Griffiths, 2007; Bowditch et al., 2018).

The Covid 19 pandemic has undoubtedly placed many individuals in difficult circumstances and meant higher levels of stress among the general population (Adams \& Schofield, 2020). The data from this experiment 
illustrated that during periods of a quarantine, socialization is an important mechanism governing gaming behaviour and choice of game. Video games can act as an important quarantine aid and escape mechanism, providing players with the ability to socialize when they are unable to leave their homes.

When comparing different traits in players, it is apparent from the data collected that although most of the participants use gaming as an outlet for stress or boredom. Many modern games rely on engaging narratives, gameplay loops, and immersive aesthetics that appeal to a player's need for socialization, creativity and immersion.

Society needs to accept video games as a powerful art form that, with proper usage, can potentially help individuals with some mental health issues. As this research has shown, video games, in particular roleplaying games, are capable of generating a strong sense of accomplishment among players. This in turn engages the players to keep coming back to the game, and leads to an increasing sense of escapism. However, care also needs to be taken to protect and help those who are using video games as a shield from the real world.

This research was undertaken during the 2020 COVID-19 pandemic. As such, the experimental data collection was limited and constrained by pandemic restrictions that were in place. Further work collecting data from a larger population sample, when restrictions are lifted, would perhaps provide further insights into gamer motivation and escapism. Further work would also act to verify the results of the work detailed in this paper.

At present, the Covid-19 pandemic continues to negatively affect society. Just as society has come to accept television and other hobbies as healthy forms of escapism during difficult times, video games too have a role to play. Even after the pandemic is over, people will always need a way to escape the issues of everyday life, and a way to socialize with far away friends. Video games will continue to be an important media form, helping players escape from the real world and helping them deal with whatever real-world stressors they are faced with.

\section{References}

Adams, J. H., \& Schofield, D. (2020). It's the End of the World and You Watch It: Media Consumption in the Time of Covid. International Journal of Contemporary Humanities, 4(1), 1-42.

Bethesda Softworks. (2012). Skyrim (Multiple Platforms) [Video Game]. Rockville, MA: Bethesda Softworks.

Blasi, M. D., Giardina, A., Giordano, C., Coco, G. L., Tosto, C., Billieux, J., \& Schimmenti, A. (2019). Problematic video game use as an emotional coping strategy: Evidence from a sample of MMORPG gamers. Journal of Behavioral Addictions, 8(1), 25-34. https://doi.org/10.1556/2006.8.2019.02

Blizzard Entertainment. (2004). World of Warcraft (PC Version) [Video Game]. Santa Monica CA: Activision Blizzard.

Bowditch, L., Chapman, J., \& Naweed, A. (2018). Do coping strategies moderate the relationship between escapism and negative gaming outcomes in World of Warcraft (MMORPG) players? Computers in Human Behavior, 86, 69-76. https://doi.org/10.1016/j.chb.2018.04.030

Calleja, G. (2010). Digital Games and Escapism. Games and Culture, 5(4), 335-353. https://doi.org/10.1177/1555412009360412

Chiu, S. I., Lee, J. Z., \& Huang, D. H. (2004). Video game addiction in children and teenagers in Taiwan. CyberPsychology \& Behavior, 7(5), 571-581. https://doi.org/10.1089/cpb.2004.7.571

Griffiths, M. D., Kuss, D. J., \& King, D. L. (2012). Video game addiction: Past, present and future. Current Psychiatry Reviews, 8(4), 308-318. https://doi.org/10.2174/157340012803520414

Kaczmarek, L. D., \& Drążkowski, D. (2014). MMORPG Escapism Predicts Decreased Well-Being: Examination of Gaming Time, Game Realism Beliefs, and Online Social Support for Offline Problems. Cyberpsychology, Behavior, and Social Networking, 17(5), 298-302. https://doi.org/10.1089/cyber.2013.0595

Kuo, A., Lutz, R. J., \& Hiler, J. L. (2016). Brave new World of Warcraft: A conceptual framework for active escapism. Journal of Consumer Marketing, 33(7), 498-506. https://doi.org/10.1108/JCM-04-2016-1775

Mathwick, C., Malhotra, N., \& Rigdon, E. (2001). Experiential value: Conceptualization, measurement and application in the catalog and Internet shopping environment11This article is based upon the first author's doctoral dissertation completed while at Georgia Institute of Technology. Journal of Retailing, 77(1), 39-56. https://doi.org/10.1016/S0022-4359(00)00045-2

Mills, D. J., Milyavskaya, M., Heath, N. L., \& Derevensky, J. L. (2017). Gaming motivation and problematic video gaming: The role of needs frustration. European Journal of Social Psychology, 48(4), 551-559. 
https://doi.org/10.1002/ejsp.2343

Normal, M. J., MdNor, K., \& Ishak, B. I. (2014). Fun beliefs in digital games from the perspective of human nature: A systematic review. In 2014 International Symposium on Technology Management and Emerging Technologies, IEEE, 359-364. https://doi.org/10.1109/ISTMET.2014.6936534

Park, J., Song, Y., \& Teng, C. (2011). Exploring the Links Between Personality Traits and Motivations to Play Online Games. Cyberpsychology, Behavior, and Social Networking, 14(12), 747-751. https://doi.org/10.1089/cyber.2010.0502

Russell, C. A., Norman, A. T., \& Heckler, S. E. (2004). The Consumption of Television Programming: Development and Validation of the Connectedness Scale. Journal of Consumer Research, 31(1), 150-161. https://doi.org/10.1086/383431

Vansteenkiste, M., \& Ryan, R. M. (2013). On psychological growth and vulnerability: Basic psychological need satisfaction and need frustration as a unifying principle. Journal of Psychotherapy Integration, 23(3), 263-280. https://doi.org/10.1037/a0032359

Wood, R. T., \& Griffiths, M. D. (2007). A qualitative investigation of problem gambling as an escape-based coping strategy. Psychology and Psychotherapy: Theory, Research and Practice, 80(1), 107-125. https://doi.org/10.1348/147608306X107881

Yee, N., Ducheneaut, N., \& Nelson, L. (2012). Online gaming motivations scale: development and validation. In Proceedings of the SIGCHI conference on human factors in computing systems, 2803-2806. https://doi.org/10.1145/2207676.2208681

\section{Copyrights}

Copyright for this article is retained by the author(s), with first publication rights granted to the journal.

This is an open-access article distributed under the terms and conditions of the Creative Commons Attribution license (http://creativecommons.org/licenses/by/4.0/). 